\title{
The RF-CV Method for Characterization of Leaky Gate Dielectrics
}

\author{
J. Schmitz ${ }^{a *}$, F. N. Cubaynes ${ }^{\mathrm{a}}$, R. de Kort ${ }^{\mathrm{b}}$, R. Havens ${ }^{\mathrm{b}}$, A. J. Scholten ${ }^{\mathrm{b}}$, and L. F. Tiemeijer ${ }^{\mathrm{b}}$ \\ aPhilips Research, Kapeldreef 75, B-3001 Leuven, Belgium \\ ${ }^{\text {b}}$ Philips Research, Prof. Holstlaan 4, 5656 AA Eindhoven, The Netherlands
}

This paper presents a new method, developed for the Capacitance-Voltage characterization of leaky dielectrics. The method comprises measurements in the gigahertz range on specially designed $\mathrm{RF}$ test structures. The method is verified against high-frequency $C$ - $V$ measurements on NMOS and PMOS structures. It yields reliable results even when the gate leakage is in excess of $100 \mathrm{~A} / \mathrm{cm}^{2}$.

\section{Introduction}

The gate current density of MOS transistors increases at an astounding pace: more than an order of magnitude per year in the last decade. Recent literature [1]-[6] discusses the difficulties encountered when a Capacitance-Voltage $(C-V)$ curve is measured on a MOS structure with high gate leakage. Typically, $C-V$ roll-off (Fig. 1) is observed at lower frequencies due to gate leakage. Given a MOS capacitance $C$ and differential conductivity $g$, a minimum measurement frequency $f_{\text {lower }}=g /(2 \pi C)$ must be used [6]. For a typical $1.5 \mathrm{~nm}$ gate dielectric with $10 \mathrm{~A} / \mathrm{cm}^{2}$ leakage, $f_{\text {lower }}=4 \mathrm{MHz}$. In recent works $[6,7]$ we therefore motivated and showed the use of radio frequency measurements to solve this problem. These papers discussed the test structure design, measurement procedure, and first results, in particular for NMOS devices in inversion. In this work, we prove the validity of RF-CV measurements on NMOS and PMOS structures for the first time. This paper also presents the first RF-CV measurements in accumulation with a leaky dielectric, and illustrates the relevance of this technique for gate dielectric research and development.

\footnotetext{
*Presently at the MESA+ Research Institute, University of Twente, P.O. Box 217, 7500 AE Enschede, The Netherlands. This research was supported by the EC within the ARTEMIS project.
}

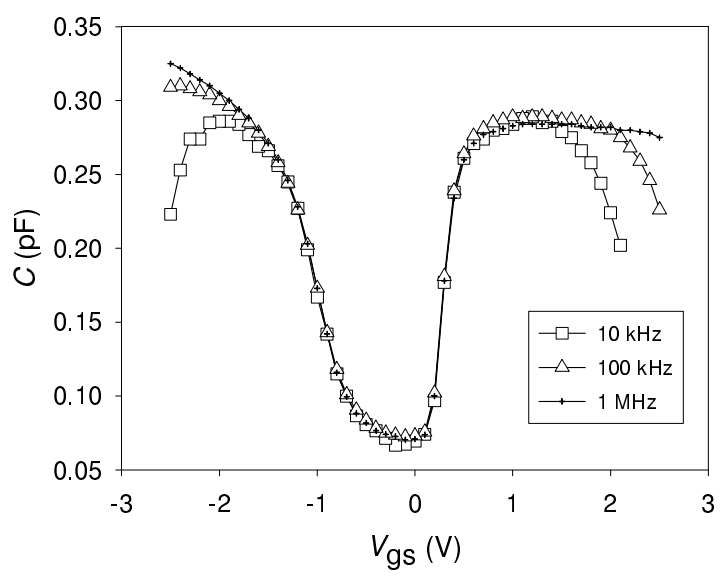

Figure 1. Typical $C$ - $V$ curves in the presence of (moderate) gate leakage. The inversion and strong accumulation parts of the curve become erroneous ("capacitance roll-off") at lower frequencies. Measurements were carried out on a W/L $=40 \mu \mathrm{m} / 0.5 \mu \mathrm{m}$ NMOS transistor.

\section{Experimental approach}

MOS capacitors and transistors were fabricated in several fabrication flows, including a 6 metal $0.13 \mu \mathrm{m}$ CMOS flow and a research process flow with high-leakage dielectrics. RF test structures, specially designed for low external resistance in inversion, depletion and accumulation [8] were tested on-wafer using an HP8510C 
VNA. $S$-parameters were measured as a function of frequency at a fixed gate bias (in the range $2.5 \ldots 2.5 \mathrm{~V})$, with the source, well and drain shortcircuited. Open-short de-embedding and parasitic resistance extraction allowed determination of the device capacitance. To obtain the capacitance in the channel region, the side capacitance (gate-drain, gate-source, and other parasitics) was effectively removed by comparison of devices with various gate lengths (see [7] for details).

\section{Validation of RF-CV measurements}

The measurement of $C-V$ characteristics requires specially designed test structures with multiple submicron gate fingers. RF capacitance measurements on such test structures are compared to classical HP4284 LCR meter measurements in Figure 2. The devices tested here exhibit only very low gate leakage, which enables such a comparison. The correspondence between the low-frequency and radio frequency results is striking. The curves overlap very well; the pointby-point relative differences are smaller than $2 \%$ in the entire range.
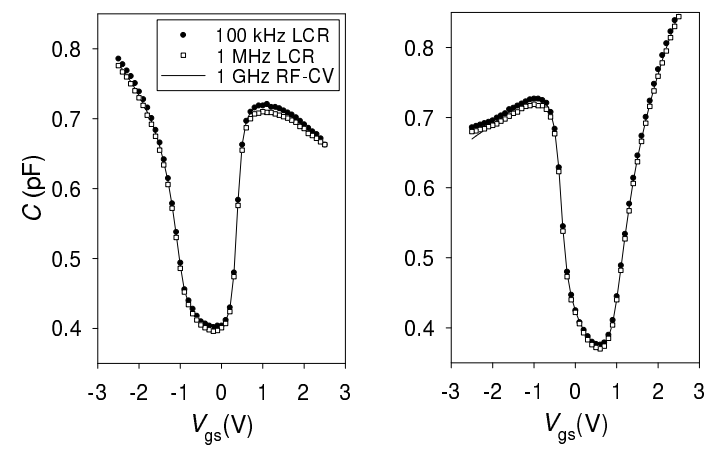

Figure 2. $C$ - $V$ measurements as obtained with an LCR meter at 0.1 and $1 \mathrm{MHz}$; and using the RF$\mathrm{CV}$ methodology, at $1 \mathrm{GHz}$. The correspondence is striking. The devices are $\mathrm{W} / \mathrm{L}=384 \mu \mathrm{m} / 0.13$ $\mu \mathrm{m}$ transistors with a low-leakage dielectric.

\section{High leakage measurements}

When a high gate leakage flows, capacitance measurement is no longer straightforward. The differential resistance of the dielectric becomes low and becomes significant with respect to the capacitive impedance $j \omega C$. This is quantified with the quality factor $Q$ of the intrinsic MOS capacitor, defined as $Q=-\operatorname{Im}(Z) / \operatorname{Re}(Z)$. When the MOS capacitor is predominantly capacitive, $Q>1$. Indeed, $Q$ should be well above unity for an accurate capacitance measurement.

The quality factor of a MOS capacitor with high gate leakage shows a strong frequency dependence, as shown in Fig. 3. The quality factor rises

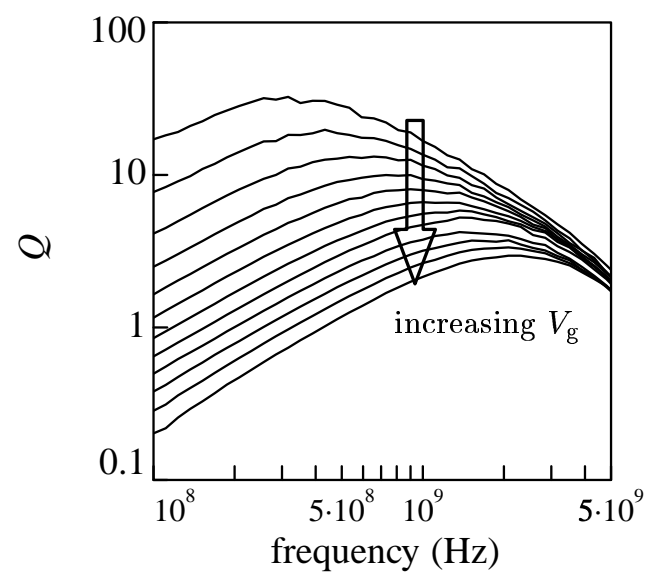

Figure 3. Measured quality factor of a $\mathrm{W} / \mathrm{L}=384 \mu \mathrm{m} / 0.18 \mu \mathrm{m}$ NMOS transistor as a function of frequency, for various gate biases in inversion $(-0.3 \ldots-1.2 \mathrm{~V})$. Severe gate leakage occurs (in particular at higher gate bias), resulting in low $Q$ below $100 \mathrm{MHz}$.

linearly in the lower frequency domain. In this regime, $Q \approx \frac{2 \pi f C}{g}$, where $f$ denotes frequency, $C$ the device capacitance, and $g$ the differential conductance of the dielectric, $g=d I / d V$. For measurements at these lower frequencies, the impedance measurement is ruled by capacitance and gate leakage. 
At higher frequencies, a maximum occurs beyond which the device becomes series resistance dominated. Here, $Q \approx \frac{1}{2 \pi f R C}$, with $R$ the external resistance in the test structure. Gate, well, source, drain and channel resistance, are the main contributors to this component (their relative significance depending on gate bias and on test structure layout).

This particular MOS device shows gate leakage between 1 and $100 \mathrm{~A} / \mathrm{cm}^{2}$ and it is clear from Fig. 3 that measurements below $100 \mathrm{MHz}$ are inadequate to obtain a good estimate of capacitance. Therefore, the normal instrumentation for $C$ - $V$ measurements, being an LCR meter with a range typically up to $1 \mathrm{MHz}$, is not applicable for investigation of dielectrics with such high leakage.

Figure 4 presents an RF-CV measurement at $0.53 \mathrm{GHz}$ of a high-leakage dielectric for the $100 \mathrm{~nm}$ CMOS node. The correct capacitance is obtained in spite of the $>100 \mathrm{~A} / \mathrm{cm}^{2}$ leakage cur-

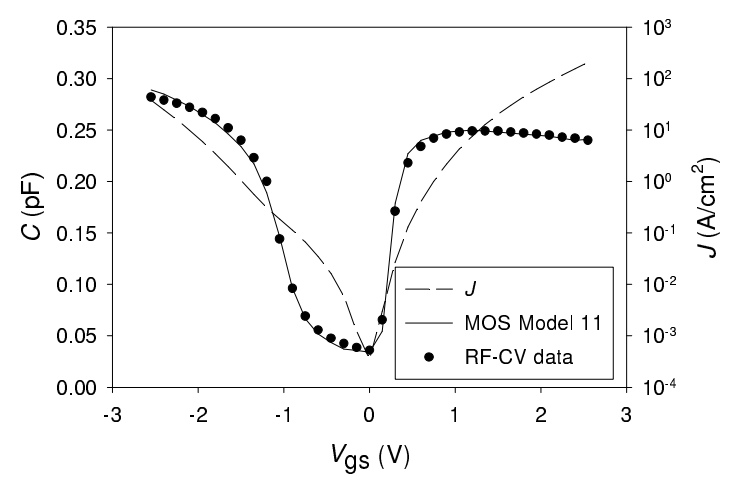

Figure 4. Intrinsic ("channel") capacitance as derived from NMOS transistors with gate leakage exceeding $10 \mathrm{~A} / \mathrm{cm}^{2}$ in accumulation and $100 \mathrm{~A} / \mathrm{cm}^{2}$ in inversion (current density $J$ : also shown). An excellent fit is obtained to MOS Model 11.

rent. This allows full $C$ - $V$ parameter extraction using a capacitance model with quantum mechanical corrections, such as MOS Model 11. In the figure, the intrinsic capacitance is plotted, defined as the capacitance of a channel segment. The overlap capacitance (gate-source and gate-drain) as well as other possible stray capacitances have been effectively removed by considering the difference between two test structures with identical layout except for the channel length $L$. From capacitance measurements, $\Delta L$ is easily retrieved, as discussed e.g. in [9]. After obtaining the effective channel length and the channel capacitance, the intrinsic capacitance can be computed and a 1D MOS model is sufficient to extract parameters from the measured $C-V$ curve.

The parameter extraction method presented here has a limited validity, and is only brought as an example. Laterally non-uniform channel doping (such as created with pocket implants), threshold voltage roll-off, and non-quasistatic effects can cause a significant error in the followed procedure. 2D models are more appropriate for $C$ - $V$ analysis of deep-submicron MOS transistors, but this track was not further pursued in the context of the present work.

At frequencies well above the optimum $Q$ (see Fig. 3) the measurements suffer from external resistance: see Fig. $5 . \quad R$ must comply with

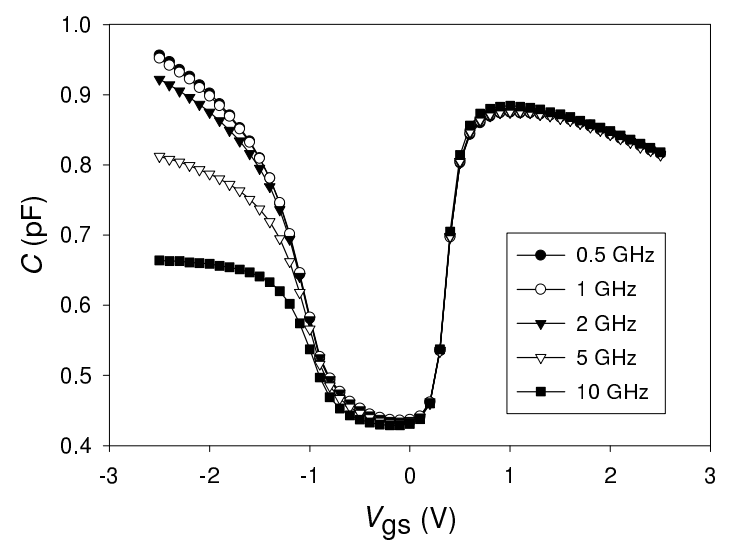

Figure 5. Typical development of the C-V curve with a measurement frequency above $Q_{\mathrm{opt}}$. The accumulation part of the curve becomes erroneous due to the RC-time in the well [7]. 
$2 \pi f R C \ll 1$ to have $Q \gg 1$. In inversion, this can be achieved by using a parallel array of shortchannel $(<1 \mu \mathrm{m})$, narrow-width $(<5 \mu \mathrm{m})$ MOS structures to suppress the resistance in the inversion layer and the gate. For RF measurements in accumulation, abundant nearby well contacts are necessary. Even then, practical limitations (gate sheet resistance, well resistance) lead to a maximum frequency in the $1-10 \mathrm{GHz}$ range.

With the RF-CV measurement approach, application of $C-V$ analysis is extended into the deca-nanometer CMOS range. Fig. 6 presents inversion $C-V$ curves of five dielectrics with very different gate leakage current densities (see inset). The dielectrics were obtained by RTO oxidation and subsequent DPN nitridation, with varying plasma exposure times. By fitting, we obtain fixed charge and equivalent oxide thickness of each of the dielectrics, supplying crucial information for process research and development [10].

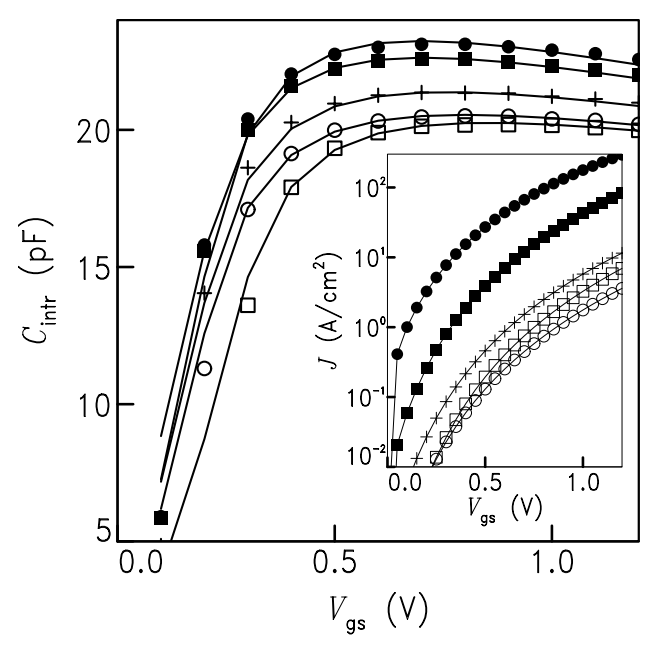

Figure 6. Inversion characteristics of NMOS capacitors with various dielectrics. Markers are measured data, lines are the MOS Model 11 capacitances. The resulting large variation in gate leakage current (inset) has no effect on the capacitance measurement.

\section{Conclusions}

Capacitance-voltage characteristics were successfully obtained from MOS capacitors with very high gate leakage densities. The RF measurement approach was found to match lower frequency $C$ - $V$ measurements in the lower gate leakage regime, for NMOS and PMOS structures. MOS parameters were successfully extracted from RF-CV curves, even at a gate leakage level exceeding $100 \mathrm{~A} / \mathrm{cm}^{2}$. The technique is effective for a wide range of test structure designs, provided the external resistance (predominantly present in the gate, inversion layer and well) is low. Applicability beyond $100 \mathrm{~A} / \mathrm{cm}^{2}$ makes the method suitable for any practical MOS technology.

\section{Acknowledgements}

The authors would like to thank D.B.M. Klaassen, Ronald van Langevelde and Johan Klootwijk for fruitful discussions.

\section{REFERENCES}

1. W. K. Henson et al., El. Dev. Lett., Vol. 20, no. 4, pp. 179-181, 1999.

2. C. H. Choi et al., El. Dev. Lett., Vol. 20, no. 6, pp. 292-294, 1999.

3. K. J. Yang and C. Hu, Trans. El. Dev., Vol. 46, no. 7, pp. 1500-1501, 1999.

4. D. W. Barlage et al., El. Dev. Lett., Vol. 21, No. 9, pp. 454-456, 2000.

5. D. W. Barlage et al., IEDM Techn. Dig., 2001, pp. 231-234.

6. J. Schmitz et al., El. Dev. Lett., Vol. 24 no. 1, pp. 37-39, January 2003.

7. J. Schmitz et al., ICMTS Conference Proceedings, 2003.

8. L. F. Tiemeijer et al., IEDM Techn. Dig., 2001, pp. 223-226.

9. R. van Langevelde et al., IEDM Techn. Dig., 2001, pp. 289-292.

10. F. N. Cubaynes et al., Proc. European ECS Conference, Paris 2003. 